\title{
Highlights
}

- The potential exists to produce new hybrid or polyploid crops in many genera

- Such new crop species may combine useful agricultural traits with hybrid vigour

- Advances in genomic technology will facilitate production of hybrid crop species 


\section{Creating new interspecific hybrid and polyploid crops}

Annaliese S. Mason ${ }^{1}$ and Jacqueline Batley ${ }^{2}$

\footnotetext{
${ }^{1}$ Plant Breeding Department, IFZ Research Centre for Biosystems, Land Use and Nutrition, Justus Liebig University, Heinrich-Buff-Ring 26-32, 35392 Giessen, Germany

${ }^{2}$ School of Plant Biology, The University of Western Australia, 35 Stirling Hwy, Crawley 6009, Australia
}

Corresponding author: Mason, A.S. (annaliese.mason@agar.uni-giessen.de)

Keywords: polyploidy, hybridization, crop improvement, sequencing, selection, meiotic stability 


\section{Abstract}

Agricultural selection of desirable traits in domesticated plant and animal species mimics natural evolutionary selection for species' ability to survive, thrive and reproduce in the wild. However, one evolutionary process is currently under-utilised for human agricultural purposes: speciation through interspecific hybridisation and polyploid formation. Despite promising successes in creation of new hybrid and or polyploid species in many genera, very few geneticists and breeders deliberately take advantage of polyploidy and interspecific hybridisation for crop improvement. We outline the possible benefits as well as potential problems and criticisms with this approach, and address how modern advances in technology and knowledge can help to create new crop species for agriculture. 


\section{A technological toolbox opening up new avenues for agriculture}

Understanding of the principles of inheritance and genetics and the successive development of new technologies [1] has carried agriculture forward to the point where an extraordinarily large number of people can be supported by a relatively small cultivated area. However, increasing population pressure, decreasing agricultural production area and climate change are now threatening food security worldwide [2]. In order to increase crop productivity, innovative new plant breeding methods are needed [2].

Plant breeding traditionally comprised simple selection and growth of plants with desirable agricultural traits. Originally, this was entirely based on phenotype: only plants which had the desired observable traits were selected to give rise to the next generation. The incorporation of the principles of genetics and heredity into understanding of crop phenotypes allowed more complex selection to take place for crop improvement [1]. Molecular markers were developed, which allowed genotypes to be inferred for some traits without relying purely on phenotypic information. This reduced the burden of phenotyping and improved the accuracy of selection, allowing breeders to more rapidly fix desirable traits in breeding pools [3]. Irradiation and related mutation-induction methodologies were also discovered and used to produce novel variation [4]. Genetic modification technology, where single gene insertions or changes are made to confer particular traits not naturally found in the crop species, shows great potential for some applications, and has successfully been used to induce disease and insect resistance and herbicide tolerance [5]. However, this methodology currently has limited application for breeding of complex, quantitative traits [6], which unfortunately includes yield and many forms of abiotic stress tolerance. Further improvements in genetic screening with the increasing accessibility of next-generation sequencing are expected to allow much more 
rigorous and rapid selection for desirable genotypes in segregating populations [6]. These advances in technology are predicted to facilitate improvement of quantitative traits like yield.

The rise of next generation sequencing has been a powerful addition to the molecular breeding toolbox. The availability of reference genome sequences for many agriculturally important species has dramatically improved our ability to identify genes of interest underlying desirable traits. This in turn allows development of molecular markers and similar genetic resources with which to characterise individual breeding lines, screen germplasm and undertake trait-related and genome-wide marker assisted selection [6]. As sequencing technology becomes ever faster and more cost-effective, genotyping-by-sequencing approaches will gradually supersede traditional approaches relying on previously developed sets of molecular markers [6].

The availability of next generation sequencing to rapidly genotype individual crop plants for selection offers a genuine breakthrough in technology. When coupled with fully annotated genomes, desirable allelic variation can be readily selected for [6]. However, fundamentally the allelic variation needs to be present in the first place before this technological leap forward can be implemented to full effect. Unfortunately, in many crop species there has been significant loss of genetic diversity, either due to intensive breeding for particular domestication traits or homogenization of breeding varieties and loss of local, adapted genotypes [7]. This is a serious issue for many of our important, staple food crops, which not only may have much slower rates of improvement through traditional selection cycles [8], but may also have shared vulnerability to particular pests and diseases as a result of inbreeding [9]. Maintaining a genetically diverse germplasm base for crop cultivars is of critical importance: the Irish potato famine, caused by late blight, and the southern corn leaf blight in the 1970s in 
the US are both examples of how serious disease outbreaks caused by over-homogenisation of crop cultivars can be [10].

Naturally, there is a solution. In the wild, plant diversity and speciation are often facilitated by interspecific hybridisation [11], and many of our important crop species are hybrids and polyploids (Box 1). Under natural evolutionary pressures, plant species often come together to form novel hybrid species. These species can benefit from increased vigour due to allelic heterosis [12], and often show transgressive segregation for phenotypic traits [13] and exploitation of different environmental niches than their progenitor species [11].

\section{Success or failure of a novel crop species is related to meiotic behaviour}

Previous attempts to produce hybrids and polyploid crops have resulted in both successes and failures. Notable small-scale successes include triticale, a hybrid between wheat and rye produced in the late $19^{\text {th }}$ century [14]; various Citrus, such as tangelo, blood lime, rangpur and limequat [15]; Prunus hybrids, such as plumcot and pluot [16], and Vitis (grapevine) hybrid varieties [17]. Many of these crops show novel phenotypes [18], as well as resistances to various pathogens [19]. However, most of our highly successful hybrid crop species (Box 1, e.g. wheat, cotton, canola, sugarcane) were formed without targeted human intervention. The reason for this is simple: most new hybrid plants are not yet genomically stable [20]. In the range of successful human-produced hybrid species listed previously, almost all are vegetatively propagated clones. This means of reproduction allows hybrids to be created and utilised without needing to have normalised meiosis and chromosome segregation. However, many novel hybrids do not initially have stable meiosis and hence regular chromosome transmission to the next generation. Without stable meiosis, these hybrids can lose chromosomes and essential genetic information, losing fertility and viability and "falling apart" within a few generations. 
In some species, such as the natural allohexaploid bread wheat, a genetic locus is present that qualitatively prevents association of non-homologous chromosomes, stabilising meiosis. In wheat, this locus is known as Ph1, for "pairing homoeologous", which it prevents between the three wheat genomes A, B and D [21]. This effect is postulated to occur by promotion of crossovers between homologues and suppression of crossovers between homeologues [22], putatively by effects on timing of chromosome condensation in chromosomes belonging to the different genomes [23]. Breeding efforts in wheat containing the Ph1 locus have allowed thousands of stable synthetic wheat varieties to be produced, incorporating valuable genetic diversity from wild progenitors [24] by utilising this natural meiotic stabilisation effect. Similarly, the effect of this locus on preventing pairing between wheat and rye chromosomes in interspecific hybrids presumably helped stabilise the novel synthetic crop triticale, a wheatrye hybrid $(2 n=A A B B R R$ or $2 n=A A B B D D R R)[25]$.

Meanwhile, in the highly agriculturally significant Brassica genus, which contains a number of vegetable (broccoli, cauliflower, buk choy, turnip) and oilseed (canola, rapeseed, mustard) crop species, similar breeding experiments have also aimed to produce novel hybrid plants. Efforts included "Raphanobrassica", a hybrid between B. napus and Raphanus [26], "Hakuran" $(2 n=$ AAAACC) [27], and allohexaploid $(2 n=$ AABBCC) Brassica [28]. However, a meiotically and reproductively stable artificial hybrid Brassica species has yet to be produced. In fact, several allotetraploid (two-genome) species already exist naturally in Brassica: oilseed rape/canola, Indian mustard and Ethiopian mustard are all allotetraploids, with genome complements $2 \mathrm{n}=$ AACC, $2 n=A A B B$ and $2 n=B B C C$ respectively [29]. Despite the existence of the diploid progenitor species $(2 n=A A, 2 n=B B$ and $2 n=C C)$, breeders and geneticists have yet to successfully recreate any of these natural hybridisation events, probably due to failure to identify and select for the quantitative allelic variation necessary to produce stable meiosis in these plants [30, 31]. The Brassica genus has no single qualitative genetic locus that "switches 
off" non-homologous chromosome pairing [30]. Widespread assessment of different genotypes of B. napus haploids has turned up only two quantitative meiotic phenotypes [32], although these results may not be generalizable to meiotic behavior in allotetraploids [33]. The major quantitative locus $\operatorname{PrBn}$ has a large effect on homoeologous pairing suppression in $B$. napus haploids [33], and several minor loci have also been identified [30]. While it is impossible to rule out a Ph1-like locus that almost completely prevents homoeologous chromosomes from pairing as homologues in Brassica, B. napus is commonly accepted to have quantitative, rather than qualitative, control of homoeologous chromosome pairing [30].

Brassica and wheat provide useful contrasting examples of success and failure (to date) of these evolutionary breeding approaches. Attempts have also been made to hybridise most other major crop species. In rice, hybrids between Oryza sativa L. and O. glaberrima Steud. have shown useful phenotypic blends of parental traits [34]. Wheat $x$ maize hybrids have been attempted, despite the large phylogenetic distance between these two species, although elimination of maize chromosomes in the zygote rather than production of hybrid plants was found to occur [35]. Although barriers to sexual hybridisation exist between many wild relatives and crop species [24], hybrids are frequently formed via rare sexual events which can be increased by some environmental stimuli [36]. Alternatively, somatic hybridisation has been successfully performed between many species and even between genera [37], and recent evidence suggests this may even be possible through grafting [38]. Regardless, there exists great potential to exploit hybrid speciation as a mechanism for crop improvement, provided meiotically stable hybrid plants can be produced.

Speed of speciation and allelic variation for meiotic behaviour 
There is increasing evidence that speciation events can be extraordinarily rapid [39]. If two species come together again to make a new hybrid species after their genomes have diverged sufficiently, very little interaction between non-homologous chromosomes is predicted [40], which would result in a more stable meiosis. Unfortunately, hybrids from divergent parents tend to be sterile, possibly as a result of increased "genome shock" and difficulties in regulating gene expression [41]. In hybrids which form between two very closely related species, it seems that the meiotic machinery can adapt rapidly to regulate meiosis and address genome stability issues, as has been demonstrated in Arabidopsis [42] and Brassica [32]. This has been suspected for some time, as there are a number of naturally occurring hybrid species that contain fundamentally un-rearranged genomes relative to the genomes of the diploid progenitors, such as recently sequenced allopolyploids wheat [43] and rapeseed [44]. Although these allopolyploid genomes show small-scale homoeologous exchanges, chromosome rearrangements accumulate in all species over time, and are thought to play a role in speciation [36]. However, genomic change in meiotically-unstable novel allopolyploids is rapid and intensive, resulting in widespread rearrangements [45]. If a substantial amount of genomic rearrangement and generational instability had occurred post-hybridisation, there would be much less collinearity between extant allopolyploids and their diploid progenitor species than is generally seen.

This leads to the question: what is the mechanism for this stabilisation after hybrid formation? Alleles contributing to meiotic stability could be inherited by chance from the parents of the first hybridisation event or concentrated through stringent selective pressures in the immediate generations after hybridisation $[42,20]$. All angiosperm species have been through multiple rounds of polyploidy [46], and in all species with more than one pair of chromosomes, some shared molecular mechanisms must exist to allow cellular recognition and distinction of homologous chromosome pairs at meiosis. This process is fundamental, and underlies all 
sexual reproduction [40]. Although there are still many unknowns, elucidation of these molecular mechanisms is rapidly progressing [36, 33]. Allelic variation operating in these molecular genetic pathways of meiotic behaviour is likely to accumulate over time, such that variation exists for stringency of control of homologue recognition and chromosome behaviour during meiosis even in diploids [20]. Recent investigation into meiotic gene evolution supports this hypothesis, suggesting that "most diploid species already have the tools to segregate chromosomes in a polyploid state" [20]. This allelic variation can be selected for during subsequent hybrid speciation events: the right combination of alleles may prevent nonhomologous chromosome pairing in the new hybrid during meiosis.

\section{Genome-wide selection for agriculturally important traits will allow rapid recovery of elite} crop phenotypes in new, stable hybrid species

Using genotyping-by-sequencing approaches, genome-wide marker assisted selection or sequence-based selection can be used to select for desirable agronomic traits [6](Box 2). This method can be utilised to retain useful alleles from wild or progenitor species [47], while maintaining "elite" cultivar status, incorporating hybrid vigour and quantifying introgressions. Genotyping-by-sequencing was recently used to precisely locate Hordeum bulbosum introgressions in the barley genome [48], and to identify numerous alleles contributing to heterosis in hybrid rice [49]. Identification of alleles related to agricultural traits has proceeded apace with developments in genotyping and sequencing technologies over the last few decades (Box 2). As fully annotated genome sequences become available for important crop species, our ability to select for agriculturally relevant and "elite" alleles underlying identified QTLs will dramatically improve [6]. The better we understand the contribution of genes and expressed sequences to phenotype, the easier it will be to build up databases of useful alleles 
and haplotype regions contributing positively to crop phenotype. Hence, new hybrids will not need to "start from scratch" in terms of crop phenotypes (Box 3).

The same methods and technological improvements will also help in meiotic stabilisation for species with poor genetic control of non-homologous chromosome interactions (Box 2). Alleles identified to contribute to meiotic stability can be similarly selected for genome-wide marker assisted selection approaches, without the need for difficult and laborious phenotyping in each generation. Approaches to effectively identify SNP molecular markers in polyploids [6] and to subsequently use next-generation sequencing to improve marker-assisted selection are now underway [50](Box 2). Large numbers of plants with different genetic backgrounds can be screened to identify alleles needed for stabilising meiosis as well as alleles related to crop yield traits. Extremely rapid and large-scale characterisation of lines using molecular techniques is now or will soon be available for many crop species [6]: this will allow breeders to catch instabilities in early generations and will aid selection for more stable lines over time. This approach will also allow maintenance of desirable genomic introgressions, selection for particular recombinants and maintenance of heterosis within individuals and populations. The more we understand and the better the technology, the more effectively we can select for optimal genome combinations to produce new crop types (Box 4).

\section{Concluding Remarks}

Formation of new hybrid and polyploid species provides a novel way forward for crop development. Recent and future advances in molecular genetics and other technologies, particularly genome sequencing and genomic selection, can help us rapidly and effectively address the problems of genome stability and retention of undesirable alleles from wild relatives. Developments in molecular genetics and genomics technology have given us the means to address the issue of genomic instability, exploiting hybrid speciation to produce 
novel crop species. Next generation sequencing technology coupled with genome annotation, increasing knowledge of biological pathways and association analysis can provide us with the ability to rapidly and effectively identify and select for desirable allelic variation. Technology can solve problems of meiotic stability in novel hybrids, allowing us to produce stable, fertile hybrid crop species for human agriculture. The potential power offered by next generation sequencing and advanced genomic approaches has major significance in addressing a biological question: how do new hybrid species form, and subsequently, how can we utilise this process for agricultural crop improvement?

\section{Acknowledgements}

ASM is funded by DFG Emmy Noether award MA 6473/1-1. JB acknowledges funding support from the Australian Research Council (Projects LP110100200, LP130100061, LP130100925, FT130100604 and DP0985953).

\section{References}

1. Moose SP, Mumm RH. Molecular plant breeding as the foundation for 21st century crop improvement. Plant Physiology. 2008;147(3):969-77. doi:DOI 10.1104/pp.108.118232.

2. Henry RJ, Nevo E. Exploring natural selection to guide breeding for agriculture. Plant Biotechnology Journal. 2014;12(6):655-62. doi:Doi 10.1111/Pbi.12215.

3. Collard BCY, Mackill DJ. Marker-assisted selection: an approach for precision plant breeding in the twenty-first century. Philos $T$ R Soc B. 2008;363(1491):557-72. doi:DOI 10.1098/rstb.2007.2170. 
4. Micke A, Donini B, Maluszynski M. Induced mutations for crop improvement - a review. Trop Agric. 1987;64(4):259-78.

5. Gasser CS, Fraley RT. Genetically engineering plants for crop improvement. Science. 1989;244(4910):1293-9. doi:DOI 10.1126/science.244.4910.1293.

6. Edwards D, Batley J, Snowdon RJ. Accessing complex crop genomes with next-generation sequencing. Theoretical and Applied Genetics. 2013;126(1):1-11. doi:DOI 10.1007/s00122-0121964-x.

7. Friedt W, Snowdon R, Ordon F, Ahlemeyer J. Plant Breeding: Assessment of Genetic Diversity in Crop Plants and its Exploitation in Breeding. In: Esser K, Lüttge U, Beyschlag W, Murata J, editors. Progress in Botany. Berlin: Springer; 2007.

8. Cowling WA. Genetic diversity in Australian canola and implications for crop breeding for changing future environments. Field Crops Research. 2007;104(1-3):103-11. doi:DOI 10.1016/j.fcr.2006.12.014.

9. Spielman D, Brook BW, Briscoe DA, Frankham R. Does inbreeding and loss of genetic diversity decrease disease resistance? Conserv Genet. 2004;5(4):439-48.

10. Cooper HD, Spillane C, Hodgkin T. Broadening the genetic base of crops: an overview. In: Cooper HD, Spillane C, Hodgkin T, editors. Broadening the genetic base of crop production. London, UK: CABI Publishing, Food and Agriculture Organizsation of the United Nations (FAO) and International Plant Genetic Resources Institute (IPGRI); 2001.

11. Ramsey J, Schemske DW. Pathways, mechanisms, and rates of polyploid formation in flowering plants. Annual Review of Ecology and Systematics. 1998;29:467-501.

12. McKeown PC, Fort A, Duszynska D, Sulpice R, Spillane C. Emerging molecular mechanisms for biotechnological harnessing of heterosis in crops. Trends in Biotechnology. 2013;31(10):549-51. doi:DOI 10.1016/j.tibtech.2013.06.008. 
13. Bell GDM, Kane NC, Rieseberg LH, Adams KL. RNA-seq analysis of allele-specific expression, hybrid effects, and regulatory divergence in hybrids compared with their parents from natural populations. Genome Biol Evol. 2013;5(7):1309-23. doi:Doi 10.1093/Gbe/Evt072.

14. Fox PN, Skovmand B, Thompson BK, Braun HJ, Cormier R. Yield and adaptation of hexaploid spring triticale. Euphytica. 1990;47(1):57-64. doi:Doi 10.1007/Bf00040364.

15. Lee LS. Citrus polyploidy - origins and potential for cultivar improvement. Aust J Agric Res. 1988;39(4):735-47. doi:Doi 10.1071/Ar9880735.

16. Topp BL, Russell DM, Neumuller M, Dalbo MA, Liu WS. Plum. Handb Plant Breed. 2012;8:571-621. doi:Doi 10.1007/978-1-4419-0763-9_15.

17. Alleweldt G, Possingham JV. Progress in grapevine breeding. Theoretical and Applied Genetics. 1988;75(5):669-73.

18. Bassene JB, Froelicher Y, Dhuique-Mayer C, Mouhaya W, Ferrer RM, Ancillo G et al. Nonadditive phenotypic and transcriptomic inheritance in a citrus allotetraploid somatic hybrid between $C$. reticulata and $C$. limon: the case of pulp carotenoid biosynthesis pathway. Plant Cell Reports. 2009;28(11):1689-97. doi:DOI 10.1007/s00299-009-0768-1.

19. Wood EA, Sebesta EE, Starks KJ. Resistance of "Gaucho" triticale to Schizaphis graminum. Environ Entomol. 1974;3(4):720-1.

20. Lloyd AH, Ranoux M, Vautrin S, Glover N, Fourment J, Charif D et al. Meiotic gene evolution: can you teach a new dog new tricks? Mol Biol Evol. 2014;In Press:doi: 10.1093/molbev/msu119.

21. Griffiths S, Sharp R, Foote TN, Bertin I, Wanous M, Reader S et al. Molecular characterization of Ph1 as a major chromosome pairing locus in polyploid wheat. Nature. 2006;439(7077):749-52. doi:DOI 10.1038/nature04434.

22. Martin AC, Shaw P, Phillips D, Reader S, Moore G. Licensing MLH1 sites for crossover during meiosis. Nat Commun. 2014;5. doi:ARTN 4580 DOI 10.1038/ncomms5580. 
23. Knight E, Greer E, Draeger T, Thole V, Reader S, Shaw P et al. Inducing chromosome pairing through premature condensation: analysis of wheat interspecific hybrids. Functional \& Integrative Genomics. 2010;10(4):603-8. doi:DOI 10.1007/s10142-010-0185-0.

24. Hajjar R, Hodgkin T. The use of wild relatives in crop improvement: A survey of developments over the last 20 years. Euphytica. 2007;156(1-2):1-13. doi:DOI 10.1007/s10681007-9363-0.

25. Lelley T, Larter EN. Meiotic regulation in triticale: interaction of the rye genotype and specific wheat chromosomes on meiotic pairing in the hybrid. Canadian Journal of Genetics and Cytology. 1980;22(1):1-6.

26. Matsuzawa $\mathrm{Y}$, Kaneko $\mathrm{Y}$, Sarashima $\mathrm{M}$. Fertility in the intergeneric hybrid plant, Raphanobrassica. Bulletin of the College of Agriculture Utsunomiya University. 1985;12(3):3140.

27. Nishi S. Differentiation of Brassica crops in Asia and the breeding of 'Hakuran', a newly synthesized leafy vegetable. In: Tsunoda S, Hinata, K., Gomez-Campo, C., editor. Brassica crops and wild allies. Tokyo: Japan Scientific Societies Press; 1980. p. 133-50.

28. Chen S, Nelson MN, Chèvre A-M, Jenczewski E, Li Z, Mason AS et al. Trigenomic bridges for Brassica improvement. Critical Reviews in Plant Sciences. 2011;30(6):524-47.

29. U N. Genome-analysis in Brassica with special reference to the experimental formation of B. napus and peculiar mode of fertilization. Japanese Journal of Botany. 1935;7:389-452.

30. Liu Z, Adamczyk K, Manzanares-Dauleux M, Eber F, Lucas M-O, Delourme R et al. Mapping $\operatorname{PrBn}$ and other quantitative trait loci responsible for the control of homeologous chromosome pairing in oilseed rape (Brassica napus L.) haploids. Genetics. 2006;174(3):1583-96. doi:10.1534/genetics.106.064071.

31. Jenczewski E, Alix K. From diploids to allopolyploids: the emergence of efficient pairing control genes in plants. Critical Reviews in Plant Science. 2004;23(1):21-45. 
32. Cifuentes M, Eber F, Lucas MO, Lode M, Chevre AM, Jenczewski E. Repeated polyploidy drove different levels of crossover suppression between homoeologous chromosomes in Brassica napus allohaploids. Plant Cell. 2010;22(7):2265-76. doi:DOI 10.1105/tpc.109.072991.

33. Grandont L, Cuñado N, Coriton O, Huteau V, Eber F, Chèvre AM et al. Homoeologous chromosome sorting and progression of meiotic recombination in Brassica napus: ploidy does matter! The Plant Cell. 2014;In Press.

34. Jones MP, Dingkuhn M, Aluko GK, Semon M. Interspecific Oryza sativa L. x O. glaberrima Steud. progenies in upland rice improvement. Euphytica. 1997;94(2):237-46. doi:Doi 10.1023/A:1002969932224.

35. Laurie DA, Bennett MD. Wheat x maize hybridization. Canadian Journal of Genetics and Cytology. 1986;28(2):313-6.

36. De Storme N, Mason AS. Plant speciation through chromosome instability and ploidy change: Cellular mechanisms, molecular factors and evolutionary relevance. Current Plant Biology. 2014;1:10-33.

37. Liu JH, Xu XY, Deng XX. Intergeneric somatic hybridization and its application to crop genetic improvement. Plant Cell Tiss Org. 2005;82(1):19-44. doi:DOI 10.1007/s11240-0046015-0.

38. Fuentes I, Stegemann S, Golczyk H, Karcher D, Bock R. Horizontal genome transfer as an asexual path to the formation of new species. Nature. 2014;In Press:doi:10.1038/nature13291. 39. Lipman MJ, Chester M, Soltis PS, Soltis DE. Natural hybrids between Tragopogon mirus and T. miscellus (Asteraceae): A new perspective on karyotypic changes following hybridization at the polyploid level. American Journal of Botany. 2013;100(10):2016-22. doi:Doi 10.3732/Ajb.1300036.

40. Bozza CG, Pawlowski WP. The cytogenetics of homologous chromosome pairing in meiosis in plants. Cytogenet Genome Res. 2008;120(3-4):313-9. doi:Doi 10.1159/000121080. 
41. Leitch AR, Leitch IJ. Genomic plasticity and the diversity of polyploid plants. Science. 2008;320:481-3.

42. Yant L, Hollister JD, Wright KM, Arnold BJ, Higgins JD, Franklin FCH et al. Meiotic adaptation to genome duplication in Arabidopsis arenosa. Curr Biol. 2013;23(21):2151-6. doi:DOI 10.1016/j.cub.2013.08.059.

43. Mayer KFX, Rogers J, Dolezel J, Pozniak C, Eversole K, Feuillet C et al. A chromosome-based draft sequence of the hexaploid bread wheat (Triticum aestivum) genome. Science. 2014;345(6194). doi:ARTN 1251788

DOI 10.1126/science.1251788.

44. Chalhoub B, Denoeud F, Liu SY, Parkin IAP, Tang HB, Wang XY et al. Early allopolyploid evolution in the post-Neolithic Brassica napus oilseed genome. Science. 2014;345(6199):950-3. doi:DOI 10.1126/science.1253435.

45. Szadkowski E, Eber F, Huteau V, Lodé M, Huneau C, Belcram H et al. The first meiosis of resynthesized Brassica napus, a genome blender. New Phytologist. 2010;186(1):102-12. doi:10.1111/j.1469-8137.2010.03182.x.

46. Jiao YN, Wickett NJ, Ayyampalayam S, Chanderbali AS, Landherr L, Ralph PE et al. Ancestral polyploidy in seed plants and angiosperms. Nature. 2011;473(7345):97-100. doi:Doi 10.1038/Nature09916.

47. Varshney RK, Nayak SN, May GD, Jackson SA. Next-generation sequencing technologies and their implications for crop genetics and breeding. Trends in Biotechnology. 2009;27(9):522-30. doi:DOI 10.1016/j.tibtech.2009.05.006.

48. Wendler N, Mascher M, Noh C, Himmelbach A, Scholz U, Ruge-Wehling B et al. Unlocking the secondary gene-pool of barley with next-generation sequencing. Plant Biotechnology Journal. 2014;12(8):1122-31. doi:DOI 10.1111/pbi.12219. 
49. Huang XH, Yang SH, Gong JY, Zhao Y, Feng Q, Gong H et al. Genomic analysis of hybrid rice varieties reveals numerous superior alleles that contribute to heterosis. Nat Commun. 2015;6. doi:ARTN 6258 DOI 10.1038/ncomms7258.

50. Yang H, Li C, Lam HM, Clements J, Yan G, Zhao S. Sequencing consolidates molecular markers with plant breeding practice. Theoretical and Applied Genetics. 2015;128:779-95.

51. Weiss-Schneeweiss H, Emadzade K, Jang TS, Schneeweiss GM. Evolutionary consequences, constraints and potential of polyploidy in plants. Cytogenet Genome Res. 2013;140(2-4):13750. doi:Doi 10.1159/000351727.

52. Lashermes $P$, Combes MC, Hueber $Y$, Severac D, Dereeper A. Genome rearrangements derived from homoeologous recombination following allopolyploidy speciation in coffee. Plant J. 2014;78(4):674-85. doi:DOI 10.1111/tpj.12505.

53. Wu Y, Sun $Y$, Wang $X$, Lin $X$, Sun $S$, Shen $K$ et al. Transcriptome shock in an interspecific F1 triploid hybrid of Oryza revealed by RNA sequencing. Journal of Integrative Plant Biology. 2015;In Press:DOI: 10.1111/jipb.12357.

54. Curk F, Ancillo G, Garcia-Lor A, Luro F, Perrier X, Jacquemoud-Collet JP et al. Next generation haplotyping to decipher nuclear genomic interspecific admixture in Citrus species: analysis of chromosome 2. Bmc Genetics. 2014;15. doi:ARTN 152 DOI 10.1186/s12863-0140152-1.

55. Tiwari VK, Wang SC, Sehgal S, Vrana J, Friebe B, Kubalakova M et al. SNP Discovery for mapping alien introgressions in wheat. Bmc Genomics. 2014;15. doi:Artn 273 Doi 10.1186/1471-2164-15-273.

56. Ruperao P, Chan CKK, Azam S, Karafiatova M, Hayashi S, Cizkova J et al. A chromosomal genomics approach to assess and validate the desi and kabuli draft chickpea genome assemblies. Plant Biotechnology Journal. 2014;12(6):778-86. doi:DOI 10.1111/pbi.12182. 
57. Snowdon R. Cytogenetics and genome analysis in Brassica crops. Chromosome research. 2007;15(1):85-95. doi:10.1007/s10577-006-1105-y.

58. Louwes KM, Hoekstra R, Mattheij WM. Interspecific hybridization between the cultivated potato Solanum tuberosum subspecies tuberosum L. and the wild species S. circaeifolium subsp. circaeifolium Bitter exhibiting resistance to Phytophthora infestans (Mont) Debary and Globodera pallida (Stone) Behrens. Theoretical and Applied Genetics. 1992;84(3-4):362-70.

59. Jones SS, Murray TD, Allan RE. Use of alien genes for the development of disease resistance in wheat. Annu Rev Phytopathol. 1995;33:429-43.

60. Zamir D. Improving plant breeding with exotic genetic libraries. Nature Reviews Genetics. 2001;2(12):983-9. doi:Doi 10.1038/35103589. 


\section{Glossary}

Allele: a DNA sequence variant of a gene, generally encoding the same protein but with slight variations. Also refers to non-coding sequence differences between two homologous DNA sequences at a single genomic location.

Allopolyploid: containing a complete set of chromosomes (haploid or diploid) from at least two different species.

Autopolyploid: containing three or more haploid sets of chromosomes from the same species (e.g. $3 x=$ autotriploid; $4 x=$ autotetraploid).

Diploid: An organism or cell containing one set of homologous chromosome pairs (two sets of chromosomes, usually of maternal and paternal origin respectively); often referred to as $2 n=$ $2 x$.

Gamete: A germline/sex cell, usually with half of the parent chromosome complement; e.g. an ovule, pollen, sperm or egg cell.

Genomic introgressions: Regions of a genome that result from recombination and integration of genetic material from one species into another species.

Genotype: The genetic information or set of allelic variants of an individual.

Genotyping-by-sequencing: Acquiring genotype information through DNA sequencing, generally via a high-throughput process.

Germplasm: a general term referring to genetic variants within a species or clade, e.g. cultivars, accessions and lines.

Haploid: A basic chromosome number set, one half of a homologous chromosome complement (often referred to as $\mathrm{n}$ or $\mathrm{x}$; the chromosome number most commonly found in gametes).

Heterosis: The effect by which two different alleles at a single genetic locus confer a greater phenotypic advantage than two copies of the same allele. 
Homologous: Containing the same sequence of genes or DNA but with (usually) different allelic variants or minor sequence variation.

Homoeologous: Sharing an ancestral homologous relationship; stretches of DNA with similarity between species or non-homologous chromosomes.

Interspecific hybridisation: When two species come together to form a new species with genetic information from both parents.

Linkage Disequilibrium: The relationship between tracts of DNA within a genome resulting from historical chromosome recombination events and population structure (e.g. selection pressure).

Meiosis: Cell division and segregation of parent chromosomes into new daughter cells to produce gametes.

Polyploidy: Presence of three or more sets of haploid chromosomes within a single cell or organism.

Phenotype: The expression of genetic information as mediated by the environment; e.g. visible traits.

Somatic: Of the body; when referring to cells those cells that are not in the germline (i.e. gametes).

\section{Box 1: Prevalence of hybrid species formation and polyploidy in crop species}

Hybrid species formation is extraordinarily prevalent in plants, and is usually accompanied by genome doubling (polyploidy), facilitated by the involvement of "unreduced gametes" (carrying a diploid rather than haploid progenitor genome) in hybridisation events [11]. Two conventional forms of polyploidy exist: allopolyploidy, which is the result of hybridisation 
between two different species, and autopolyploidy, which is the result of duplication of a single genome, or possibly hybridisation between two different genotypes within the same species. Auto- and allopolyploidy exist on a spectrum rather than being discrete from each other, as the relationship between the progenitors can vary from very distant (e.g. species from two different genera) to autopolyploids arising from a single plant [11]. However, allopolyploidy is perhaps of greater interest for breeding than autopolyploidy, as allopolyploidy generates more novel genetic diversity and hybrid vigour [13] and generally (although not always) results in less meiotic instability $[51,11]$. All angiosperm species have at least one historical polyploidy event [46], and many genera have undergone several rounds of hybridisation and genome doubling in fairly recent history [41]. Many of our modern crop species are polyploid, such as potato (Solanum), sweet potato (Ipomoea), cassava (Manihot), taro (Colocassia) and yams (Dioscoria), wheat (Triticum), sugarcane (Saccharum), cotton (Gossypium), canola (Brassica), strawberry (Fragaria) and kiwifruit (Actinidia). Other crops are interspecific hybrids, such as most citrus cultivars [15] and domesticated apples, while other crops are triploids, such as banana and seedless watermelon.

\section{Box 2: Developing genomics tools to analyse polyploids and interspecific hybrids}

Next generation sequencing has already been demonstrated to have use in understanding the genomes of polyploid crop species. RNASeq data has been used to quantify genomic changes occurring as a consequence of polyploidisation in coffee [52]: homoeologous exchange events shared by all accessions confirmed that a single polyploidisation event had occurred. RNASeq was likewise used to investigate "transcriptome shock" in interspecific rice hybrids [53]. The genomes of species which have originated via natural interspecific hybridisation, such as many citrus crops, can also be characterised to identify the chromosomal fragments that are 
contributed by the different ancestral genomes. This allows the identification of desirable (and undesirable) traits, and can be used for optimising breeding, as shown using 454 sequencing [54].

The ability to isolate chromosomes through flow sorting has revolutionised understanding of crop genome structure. Whilst the technology has previously been limited to sorting chromosomes by size, the future possibility of sorting single chromosomes will enable the identification and quantification of introgressions in any species. Chromosomes isolated by flow sorting have been used to identify alien introgressions in wheat [55], and to determine the difference between sequence errors and true structural variation between desi and kabuli chickpea types [56]. Similar advances and technological developments, particularly in the area of next-generation sequencing, are continually advancing our understanding of the processes of polyploidy and interspecific hybridisation, and allowing detailed analysis of polyploid and hybrid crop genomes.

Box 3. Exploiting genetic diversity in wild relatives through genomic introgressions into crop species

Plant breeders have long been aware of the power of hybridisation between species for agricultural improvement. In fact, introgressive breeding, whereby a novel phenotypic or allelic variant in a wild or related species is crossed into a crop species through hybridisation followed by successive backcrossing, has been carried out in a number of crop species to great effect. In canola (Brassica napus), hybridisation with related species B. rapa, B. juncea and B. carinata has resulted in transfer of resistance to Verticillium longisporum (Verticillium wilt) and Leptosphaeria maculans (blackleg) [57]. Cultivated potato (Solanum tuberosum) has also 
benefited from interspecific hybridisation, resulting in transfer of resistance to Phytophthora infestans and Globodera pallida [58], among others. In wheat, resistance to barley yellow dwarf virus, wheat streak mosaic virus, Cephalosporium stripe and eyespot has been introgressed into the crop from wild grass species [59].

Incorporating genetic diversity from wild relatives has already played a key role in crop improvement, particularly with respect to disease resistance and abiotic stress tolerance [57, $24,60]$. We suggest that the mechanism of interspecific hybridisation can be extended from its current uses in genomic introgression to production of novel crop species. Producing a new species, especially a hybrid, is often perceived to involve starting again from scratch in terms of desirable agronomic traits. Outcrossing elite breeding material can result in loss of quality and yield traits, disrupting linkage disequilibrium blocks and introducing unfavourable alleles [60]. Thus, despite the established advantages to be gained from incorporation of additional genetic diversity and useful traits from wild progenitors into crop germplasm [24], and from the further leap to production of new crop species, this approach has previously been littleexplored for crop development. However, we propose that with greater understanding of genomes and trait inheritance, and with the further development of genotyping by sequencing technology, recovery and build-up of desirable crop phenotypes in novel hybrid species is achievable.

\section{Box 4. Outstanding Questions}

Exactly which genetic and genomic factors contribute to meiotic stability and to important crop phenotypes in species and genera of agricultural interest? How can we best utilise knowledge of these factors and optimise selection methods to facilitate breeding of new, 
stable hybrid crop species? In which genera can we best take advantage of interspecific hybridisation and/or polyploidy processes to develop new crop types? 